\title{
IMPLEMENTATION OF FLEX SENSOR FOR HAND GESTURE BASED WIRELESS AUTOMATION OF JCB MACHINE
}

\author{
Kapil R. Dhanwani ${ }^{1}$, Jayesh P. Gindani ${ }^{2}$ \\ ${ }^{I}$ Kapil Dhanwani, Electronics \& Communication, S.V.I.T, Gujarat, India \\ ${ }^{2}$ Jayesh Gindani, Electronics \& Communication, S.V.I.T, Gujarat, India
}

\begin{abstract}
The project is totally based on automation. It has been made to contribute in the field industry. Many Projects have been undertaken previously to develop automatic JCB using various technology like Remote control, Zig bee control etc. But none have such Dual Mode feature like Flex Sensor control and DTMF control with LDR sensor, Flame Detector and IR sensor integrated on a single chip. This project will not require a worker sitting inside a JCB and can be run from outside with more comfortable and more accuracy and is also cost effective and requires low maintenance. It can be controlled by simple hand gestures. It employs a Flex sensor which controls the JCB's movements like up-down and 360 degree clockwise and anticlockwise rotation by bending it and making our JCB move accordingly. Also if any problem occur in Flex Sensor then we can switch to other mode of operation which is DTMF. Using which we can easily control our JCB worldwide through cell phone. The entire core of the project lies in the microcontroller which is a device used to establish automation. The main aim of this paper is to implement a JCB with Dual mode of operation. It also includes other features like IR sensor to detect any obstacle in field work like excavation, LDR sensor to make our JCB work at night also by automatically illuminating the LED's in the dark places. Flame Detector to detect fire when any kind of accident occur.
\end{abstract}

Key Words: Flex sensor, DTMF control, IR sensor, Flame detector etc... $* * *$

\section{INTRODUCTION}

Robotics is best suited for industrial automation like manufacturing, packaging, picking up heavy objects. Most of the tasks in these sectors are dangerous for human beings and as such utilizing Robotics and automation in these sectors would improve productivity, safety as well as the quality. Our project aims at developing a wireless JCB machine to help out field industries. The Machine will moves and can be used for excavation and drilling depending upon different moves perfomed by users. Apart from this, it has two modes of operation so that if one fails the other one can be used and we can continue our field work and any mode can be used any time by just triggering a switch and they are:-

\subsection{Flex sensor Mode}

Flex sensors known as bend sensors are analog resistors. The property of flex sensor is that when we bend a sensor it produces resistive output which is related to bend radius. When the radius is small the output will be high. The higher the variation in deflection or bending of flex sensor results in higher variation of resistance itself. Potentiometer is used to read these resistance changes and it is given to ADC which converts these values into equivalent digital values and fetched it into RF module through which it gets transmitted.

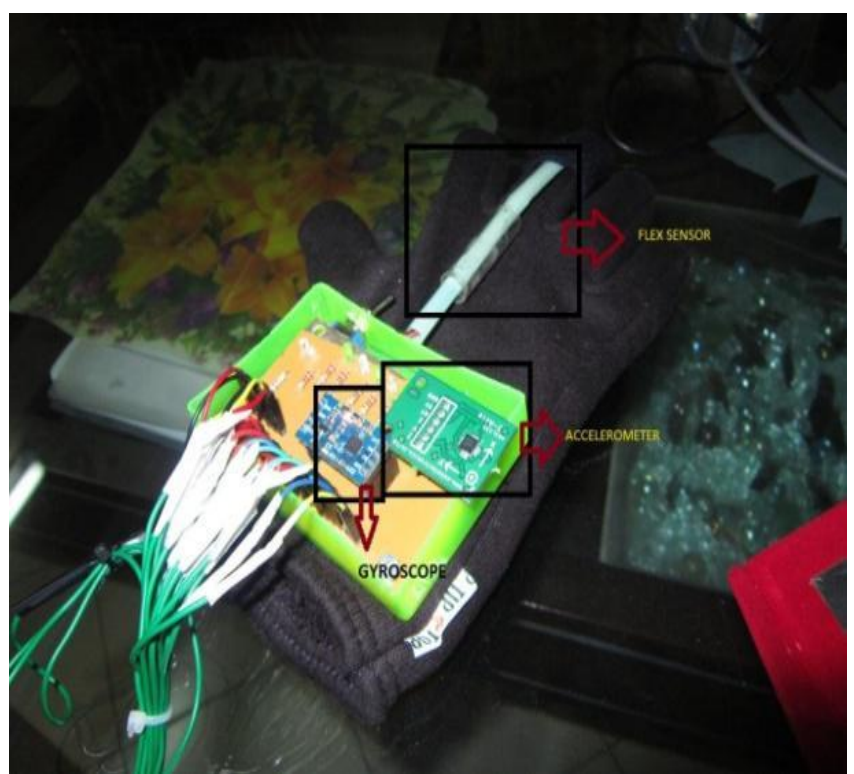

Fig-1: FLEX SENSOR

\subsection{DTMF Control Mode}

DTMF stand for Dual Tone Multiple Frequency. DTMF signal is generated when we press any of keys on keypad of telephone. With each key pressed the generated DTMF signal is the amplitude modulation of two frequency. From which one frequency is of higher value and the other one is of lower value. Both these frequencies under goes analog modulation and merge it into a single frequency and gets transmitted using transmitter. 


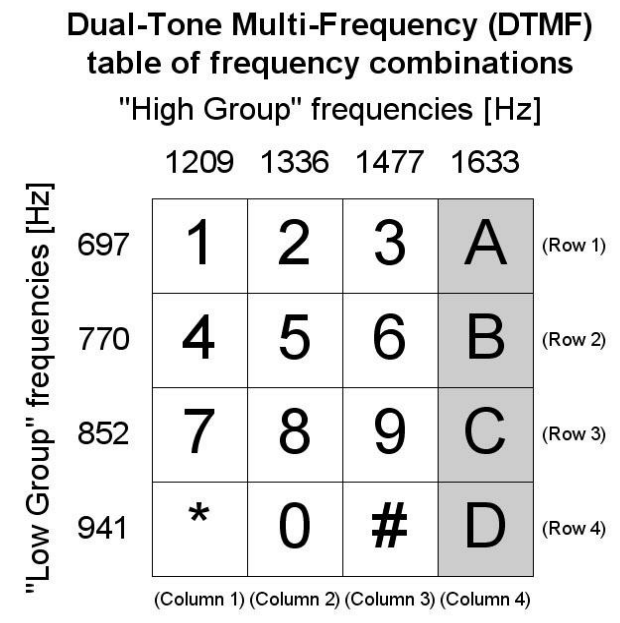

Fig-2: DTMF

\section{METHODOLOGY}

\subsection{Obstacle Sensing:}

It consist of IR transmitter, TSOP receiver and IC LM358 as a major components. While moving on the track, IR emits infra red light in forward direction and in case if some obstacle is present on the way of moving the reflection of infrared light is receive by TSOP receiver sensor which amplified the weak signal and give the output in $36 \mathrm{khz}$ frequency which are connected on the interrupt pin of the controller which enable immediate stopping of all the operation and vehicle stop while interrupt disable when object is not present in front of the vehicle, no reflection will come and vehicle will perform its programmed functions.

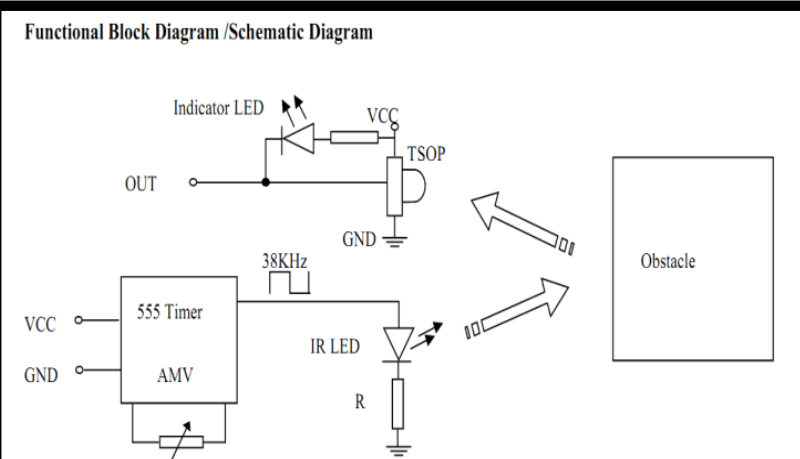

Fig -3: Mechanism of obstacle sensing

\subsection{RF module:}

A Radio Frequency module is a small electronic device used to transmit and receive radio signals between two devices. In every system, RF module is used to communicate wirelessly. The frequency range varies between $30 \mathrm{kHz}$ \& $300 \mathrm{GHz}$. The data or signals are represented as variations in the amplitude of carrier wave. The modulation used in $\mathrm{RF}$ is known as Amplitude Shift Keying (ASK). This RF module comprises of an RF Transmitter and an RF Receiver. An RF transmitter receives serial data and transmits it wirelessly through its antenna. Transmission through RF is better. Signals through RF can travel to larger distance making it suitable for long range applications. Normally IR can operate properly only in line-of-sight mode, while RF signals can travel even when there is an obstacle between transmitter \& receiver.

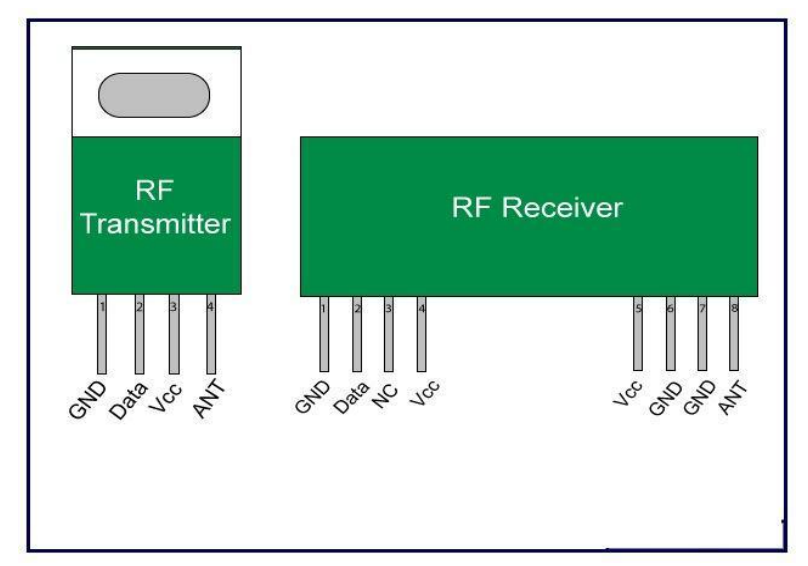

Fig -4: RF Module

\subsection{Motors:}

\section{Why DC Motors?}

The dc motors are most easy to control .One dc motor will require only two dc signals for its operation, if we want to change the direction then we just need to change the polarity of the power across it. We can vary speed by varying the voltage across the motor by making use of gears as gears increases the torque at the expense of the speed.

Mathematical interpretation:-

$$
\begin{aligned}
& \text { Rotational power }(\mathrm{P})=\text { Torque }(\mathrm{T}) * \text { Rotational speed }(\mathrm{S}) \\
& \Rightarrow \quad \mathrm{T}=\mathrm{P} / \mathrm{S}
\end{aligned}
$$

$\mathrm{P}$ is constant for dc motor with constant electrical power. Thus the torque is inversely proportional to the speed

$$
\mathrm{T} \text { a } 1 / \mathrm{S}
$$

By using two motors we can move robot in any direction.

\section{Usage of motor driver:}

From microcontroller we cannot connect a motor directly because microcontroller cannot give sufficient current to drive the dc motor. The motor drive connected across it is a current enhancing device which act as a switch. Thus we insert a motor drive between motor and microcontroller. Using our L293D H-Bridge based product, one can easily drive relays, solenoids, DC and step-motors. It has 4 control lines which allow you to drive forward, backward and stop.

\section{L293D Logic Table:}

Pin2 =Logic 1 and Pin7=Logic $0 \mid$ Clockwise Direction.

Pin2 =Logic 0 and Pin7=Logic $1 \mid$ Anticlockwise Direction.

Pin2= Logic 0 and Pin7= Logic $0 \mid$ Idle [No rotation] [Hi-Impedance state].

Pin2 = Logic 1 and Pin7 = Logic $1 \mid$ Idle [No rotation]. 


\section{Clockwise rotation}

: Fig -5Anticlockwise rotation:

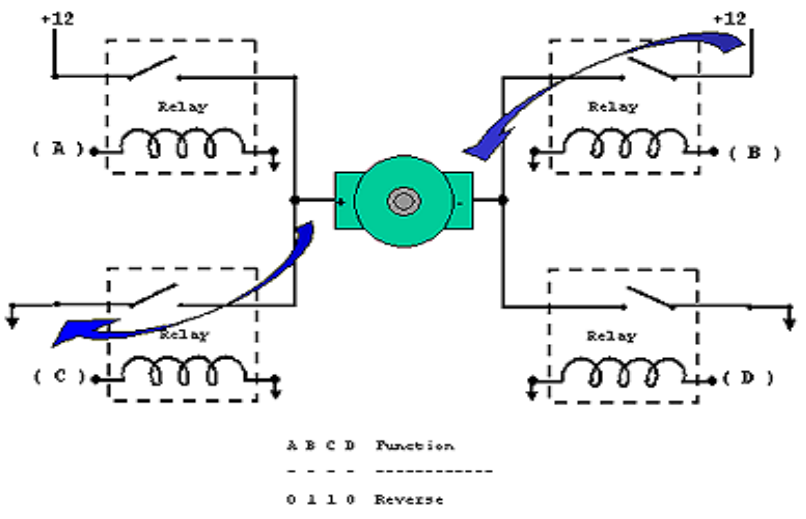

Fig -6

\subsection{Dark Room Detector:}

A dark room detector is a variable resistor whose value changes depending on incident light intensity.

Table-1: Specifications of LDR

\begin{tabular}{|l|l|}
\hline PARAMETERS & VALUE \\
\hline Voltage(Peak AC or DC) & $250 \mathrm{~V}$ \\
\hline Operating temperature(deg C) & -60 to 75 \\
\hline Power dissipation(at $30 \mathrm{deg}$ C) & $250 \mathrm{~mW}$ \\
\hline
\end{tabular}

The LDR is connected to the LCD in the potentiometer configuration so as to give the microcontroller the analog signal in voltage form. It works on the basis of Transistor Transistor Logic(TTL). When light falls on LDR, its resistance decrease which drives transistor Q1-BC547 into conduction mode. This keeps transistor Q2 in cut-off mode. The LED does not glow as long as light falls on LDR. When the resistance of LDR becomes high during night time, transistor Q1 stops conducting and transistor Q2 starts conducting to turn on the LED. The use of LDR sensor in our project is to turn on automatically the light system during night time so that our JCB can work in night time also.

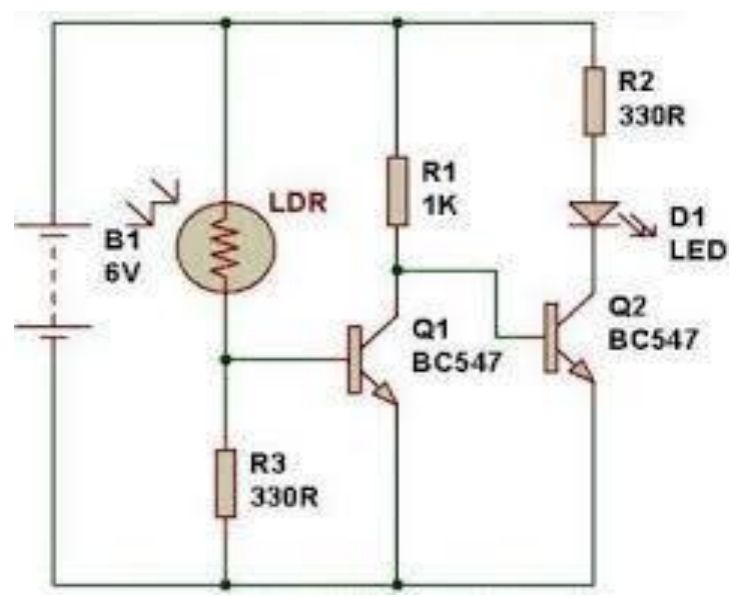

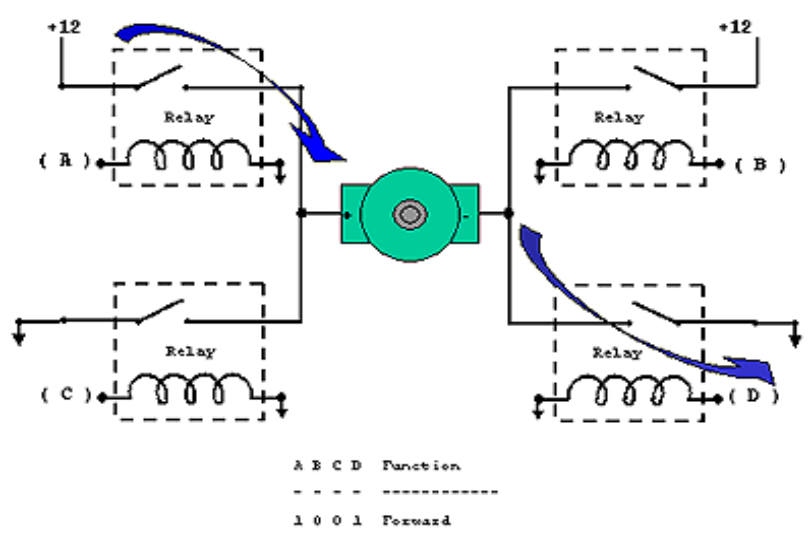

Fig-7: Mechanism of LDR Sensor

\subsection{Microcontroller Arduino Uno:}

An Arduino is open source physical computing platform based on a simple microcontroller board.

Table-2: Specifications of Arduino

\begin{tabular}{|l|l|}
\hline PARAMETERS & VALUES \\
\hline Microcontroller & ATmega328 \\
\hline Operating Voltage & $5 \mathrm{~V}$ \\
\hline Input Voltage (recommended) & $7-12 \mathrm{~V}$ \\
\hline Input Voltage (limits) & $6-20 \mathrm{~V}$ \\
\hline Digital I/O Pins & 14 \\
\hline Analog Input Pin & 6 \\
\hline DC Current for 3.3V Pin & 40 \\
\hline Flash Memory & 32 \\
\hline SRAM & $2 \mathrm{~KB}(\mathrm{ATmega328})$ \\
\hline EEPROM & $1 \mathrm{~KB}(\mathrm{ATmega328})$ \\
\hline Clock Speed & $16 \mathrm{MHz}$ \\
\hline
\end{tabular}

To make our project work, the heart of the project remains ATMEGA 328 controller wherein program is fed to automate the product. These programs have been made using specific software like EMBEDDED C. Thus features of the software helps us to execute the steps of implementation and provide an environment to actually implement the innovation thought of.

\section{DESIGN ANALYSIS}

\section{Observation matrix canvas}

The entire model is divided into four modules.

\section{FIRST MODULE: OBSERVATIONS:}

Observations that we can make with this project so that we can correlate it with other things like Automation, Robotics, Embedded, Excavation Robot, Wireless network etc.

\section{SECOND MODULE: SCOUTED CHALLENGES:}

Here we are dealing with the challenges that we are facing in our project such as Software programming, small scale, Compatibility problem, Sources issue, cost etc.

Among this the most important challenges that we are facing is image Quality, time bound \& Battery Drain. 


\section{THIRD MODULE: TOP 5 PROBLEM ON THE BASIS OF DESIRABILITY,FEASIBILITY AND VIABLITY;}

\section{1) Software}

Particular coding of flex sensor

2) Sensors

Digital protocol dependent

3) Interfacing

Interfacing of various parts

4) Battery Drain

Power consumption by JCB

5) Operation Modes

DTMF and Flex Sensor

\section{FOURTH MODULE: SELECT 1 FROM TOP 5; OPERATION MODE}

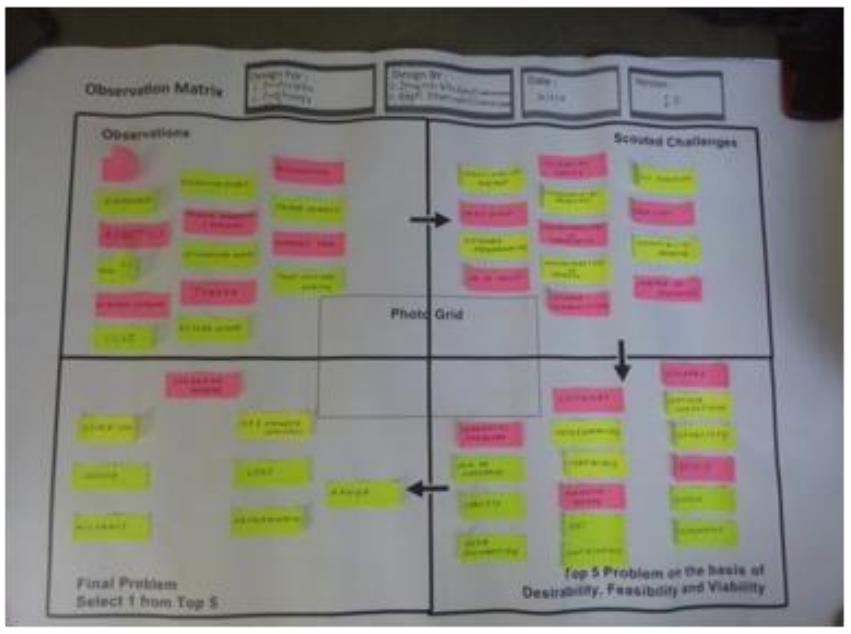

Fig-8: Observation canvas

\section{CONCLUSIONS}

This project has been made for excavation in field industry without human labor. Automatic picking of heavy objects and placing it at other different location in field industries. Automatic breaking and crunching of heavy rocks which are located in high temperature zones. The operator can control the whole JCB functions from outside the hazardous areas by looking through a glass door. With simple movements of gesture, users can easily control connections between multiple devices.

Also it can work through its other mode of operation also which is DTMF when the failure of Flex sensor occurs from anywhere by just establishing a cellular connection. By this model, accuracy of excavation increases and accidents decreases. Thus, as described above we can say that the implementation of our module is very easy to understand, more user friendly, cheaper in cost, and easy algorithm of working

\section{Future Scope}

The application implemented by the present model is just a prototype which can be extended by adding many other features into it as per requirement. Thus model has been kept open for the addition of other features whose need would be felt in the near future and wherein more automation would be added. The system can further be improved by replacing the CPU that is the Arduino ATMEGA328 microcontroller to development platforms like raspberry pi, beagle bone, etc. where image processing can further be done separately avoiding any human engagement in monitoring. Human detection sensor can be used to increases the safety of human being. When human comes in the range of sensor buzzer will ring to indicate the human interface.

\section{REFERENCES}

[1] Thomas G. Zimmerman, Flushing, N.Y for Ineractive Gesture Interface by Flex Sensor.

[2] Pierre Sauvage, Notre Dame de Commiers(FR), Mark Brandt, Robert Hyerle for DTMF Control.

[3] Willem Den Boer, Hillsboro, OR(US), Shahram Izadi Cambridge (GB) for Obstacle detection.

[4] Hong Fu Gin Precision Industry, Tu Cheng(TW) for Robotic arm.

\section{BIOGRAPHIES}

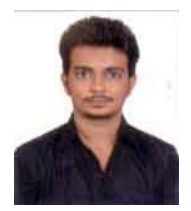

Kapil R. Dhanwani

BE(Electronics \& Communication)

Sardar Vallabhbhai Patel Institute of Technology

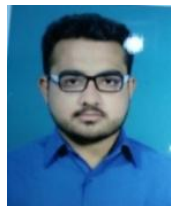

\section{Jayesh P. Gindani}

BE(Electronics \& Communication)

Sardar Vallabhbhai Patel Institute of Technology 